MIDPI

MOL2NET, International Conference Series on Multidisciplinary Sciences

sciforum

\title{
Ecotoxicological risk assessment and prioritization of pharmaceuticals using QSTR approaches
}

Kabiruddin Khan ${ }^{a}$ (kabirkhan78621@ gmail.com), Emilio Benfenati ${ }^{b}$

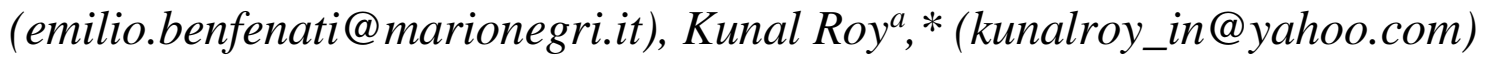

${ }^{a}$ Pharmaceutical Technology, Jadavpur University, 188 Raja S C Mullick Road, 700032, Kolkata, India

${ }^{b}$ Laboratory Of Environmental Chemistry And Toxicology, Irccs - Istituto Di Ricerche

farmacologiche Mario Negri, Via La Masa, 19, 20156, Milano, Italy

*Corresponding author.

\section{Graphical Abstract}

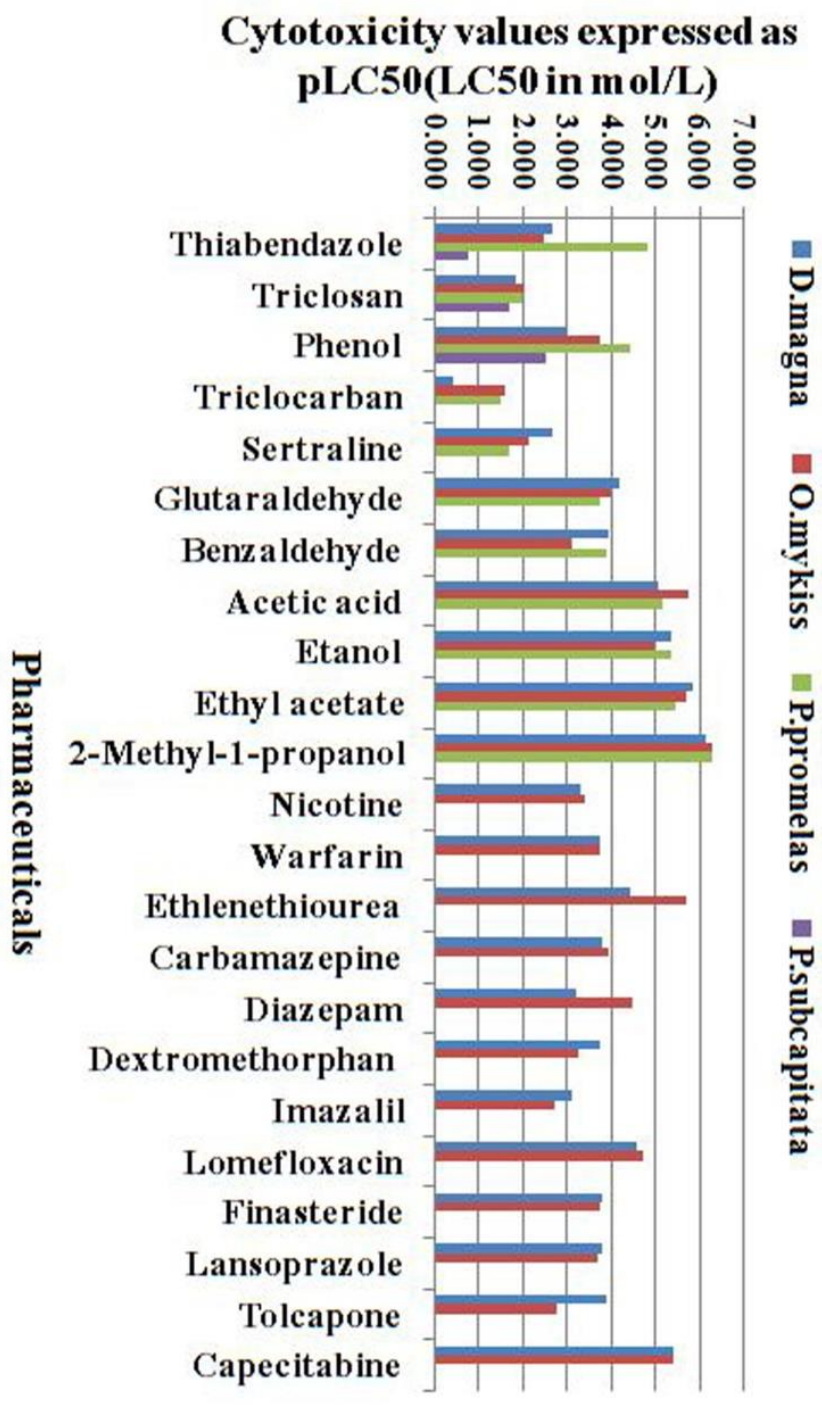

\section{Abstract}

The occurrence and risk associated with pharmaceuticals in aquatic flora is insufficiently understood due to a huge lack of documented resources. However, in recent decades, an enhanced focus has been made towards testing of the level of pharmaceuticals in water bodies due to several reports on accumulation of these ingredients to the range of not less than $0.1 \mu \mathrm{g} / \mathrm{L}$ in surface waters. Due to involvement of time, cost, labor and animal sacrifice associated with experimental testing of toxicity, Quantitative Structure-Toxicity Relationship (QSTR) models have been recommended to bridge data gaps by European Union Commission's Scientific Committee on Toxicity, Ecotoxicity, and Environment (CSTEE). In the current work, QSTR models have been developed for the toxicity of pharmaceuticals against Pseudokirchneriella subcapitata, Daphnia magna, Oncorhynchus mykiss and Pimephales promelas. The acute experimental toxicity data were mainly taken from the ECOTOX database. Genetic algorithm followed by partial least squares technique was used for model development whereas validation was performed using stringent internal and external validation metrics, namely cross-validated leave-oneout predicted variance for the training set while average rm2test and MAE95\% for the test set, following strict OECD guidelines. Selected 2D descriptors namely extended topochemical atom 
(ETA) descriptors, constitutional indices, functional group count, atom-centered and CATS-2D were calculated from DRAGON and PaDEL-Descriptor software for model development. The frequent repetition and higher VIP value of $2 \mathrm{D}$ atom pair descriptors namely (B01[C-X], F08 [O-O]) suggest their significant impact on toxicity of pharmaceuticals against different aquatic organisms followed by the number of rotatable bonds and presence of lone pair electrons involved in resonance with aromatic structures as defined by the 2nd generation ETA indices. Models were developed with and without LogP/lipophilicity descriptors separately to understand the impact of $\log \mathrm{P}$ on the model quality. The applicability domain study was carried out using different techniques namely DModX and standardization approach in order to set a predefined chemical zone of applicability for the obtained QSTR models; the external test set compounds falling outside the domain were not taken for further analysis while making a prioritized list of most potent emerging contaminants. An additional comparison was made with predictions from ECOSAR, an online expert system, using calculated RMSE values obtained fromour models and those calculated by ECOSAR, in order to prove predictability of the obtained models. Finally, the obtained robust models were utilized to rank a large dataset of approximately 1267 drug like molecules in order of their potential for emerging contaminants following a scaling technique.

\section{References}

[1] Roy, Kunal, Supratik Kar, and Rudra Narayan Das. Understanding the basics of QSAR for applications in pharmaceutical sciences and risk assessment. Academic Press, NY, 2015.

[2] https://cfpub.epa.gov/ecotox/

[3] http://teqip.jdvu.ac.in/QSAR_Tools/DTCLab/ 